OPEN ACCESS

Edited by:

Olga Matos,

New University of Lisbon, Portugal

Reviewed by:

Fernando Izquierdo Arias, CEU San Pablo University, Spain

Maria Luisa Lobo,

New University of Lisbon, Portugal

*Correspondence:

Meng Q

qimengdz@163.com

Long-Xian Zhang

zhanglx8999@henau.edu.cn

tThese authors have contributed equally to this work

Specialty section: This article was submitted to

Infectious Diseases,

a section of the journal

Frontiers in Microbiology

Received: 19 August 2019 Accepted: 04 October 2019

Published: 22 October 2019

Citation:

Li D-F, Zhang $Y$, Jiang $Y-X$, Xing J-M, Tao D-Y, Zhao A-Y, Cui Z-H, Jing B, Qi M and Zhang L-X (2019) Genotyping and Zoonotic Potential of Enterocytozoon bieneusi in Pigs

in Xinjiang, China.

Front. Microbiol. 10:2401. doi: 10.3389/fmicb.2019.02401

\section{Genotyping and Zoonotic Potential of Enterocytozoon bieneusi in Pigs in Xinjiang, China}

\author{
Dong-Fang Li ${ }^{1,2+}$, Ying Zhang ${ }^{1 \dagger}$, Yu-Xi Jiang ${ }^{1}$, Jin-Ming Xing ${ }^{1}$, Da-Yong Tao',

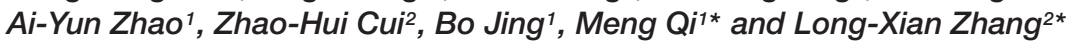 \\ ${ }^{1}$ College of Animal Science, Tarim University, Alar, China, ${ }^{2}$ College of Animal Science and Veterinary Medicine, Henan \\ Agricultural University, Zhengzhou, China
}

Enterocytozoon bieneusi is an obligate intracellular fungus, infecting various invertebrate and vertebrate hosts, it is common in humans and causes diarrhea in the immunocompromised. In the present study, 801 fecal specimens were collected from pigs on seven large-scale pig farms in Xinjiang, China. Nested polymerase chain reaction (PCR) amplification of the internal transcribed spacer (ITS) gene showed that the overall E. bieneusi infection rate was $48.6 \%$ (389/801). The E. bieneusi infection rates differed significantly among the collection sites (20.0-73.0\%) $\left(x^{2}=75.720, d f=6, p<0.01\right)$. Post-weaned pigs had the highest infection rate $(77.2 \%, 217 / 281)$, followed by fattening pigs $(67.4 \%, 87 / 129)$ and pre-weaned suckling pigs $(35.5 \%, 60 / 169)$. Adult pigs had the lowest infection rate $(11.3 \%, 25 / 222)$. The E. bieneusi infection rates also differed significantly among age groups $\left(x^{2}=246.015, d f=3, p<0.01\right)$. Fifteen genotypes were identified, including 13 known genotypes (CHC, CS-1, CS-4, CS-7, CS-9, D, EbpA, EbpC, EbpD, H, PigEb4, PigEBITS5, and WildBoar8) and two novel genotypes (XJP-\| and XJP-III). Among them, six genotypes (CS-4, D, EbpA, EbpC, H, and PigEBITS5) have been reported in humans. Phylogenetic analysis showed that all the genotypes belonged to Group 1 of $E$. bieneusi. These findings suggest that pigs may play an important role in transmitting $E$. bieneusi infections to humans.

Keywords: Enterocytozoon bieneusi, infection rate, novel genotype, potential zoonotic, pig

\section{INTRODUCTION}

Over 1300 microsporidial species infect a variety of invertebrate and vertebrate hosts, Enterocytozoon bieneusi is considered the most common microsporidial species to cause opportunistic infections in humans (Sak et al., 2011; Matos et al., 2012). E. bieneusi infections cause diarrhea, malabsorption and possible lung pathologies, and host health status is the main influencing factor (del Aguila et al., 1997; Matos et al., 2012). E. bieneusi is transmitted mainly via the fecal-oral route through ingestion of contaminated water or food or accidental ingestion of spores eliminated in the feces of infected animals or humans (Stentiford et al., 2016). 
Analysis of the ribosomal internal transcribed spacer (ITS) nucleotide sequence polymorphism is widely used for E. bieneusi molecular typing (Santín and Fayer, 2011; Zhang et al., 2011; Karim et al., 2014; Liu et al., 2017; Deng et al., 2018). Over 470 E. bieneusi genotypes have been identified from the ITS gene in humans, mammals, birds and water; however, this pathogen also exists in many undefined areas worldwide (Henriques-Gil et al., 2010;
Li D. et al., 2019). Phylogenetic analysis has revealed high diversity and genetic variation among isolates from human and animal origins, and these isolates are clustered into 11 major genetic groups (Galván-Díaz et al., 2014; Wang et al., 2019).

Studies have identified zoonotic E. bieneusi genotypes from pigs in China, thus implicating pigs as dispersing agents and a potential source of human infections (Li et al., 2014a,b, 2017;

TABLE 1 | Enterocytozoon bieneusi occurrence and genotype distribution in pigs in Xinjiang, China.

\begin{tabular}{|c|c|c|c|}
\hline Collection site & No. positive/No. specimens & $\%(95 \mathrm{Cl})$ & Enterocytozoon bieneusi genotypes (n) \\
\hline Marabishi & $48 / 98$ & $49.0(38.9-59.1)$ & CHC5 (1), CS-7 (3), D (6), EbpA (1), EbpC (34), EbpD (1), PigEBITS5 (2), \\
\hline Alaer & $19 / 95$ & $20.0(11.8-28.2)$ & $\mathbf{D}(1), \boldsymbol{E b p C}(17), \mathbf{H}(1)$ \\
\hline Yarkant & $63 / 130$ & $48.5(39.8-57.2)$ & CHC5 (1), D (8), EbpA (43), EbpC (9), PigEBITS5 (2), \\
\hline Baicheng & $67 / 99$ & $67.7(58.3-77.1)$ & EbpA (50), EbpC (10), PigEBITS5 (7) \\
\hline Shaya & $73 / 100$ & $73.0(64.1-81.9)$ & $\begin{array}{l}\text { CS-1 (3), CS-4 (20), CS-9 (1), EbpA (11), EbpC (11), EbpD (3), PigEb4 (12), } \\
\text { PigEBITS5 (6), WildBoar8 (3), XJP-II (2), XJP-III (1) }\end{array}$ \\
\hline Changji & $49 / 130$ & $37.7(29.3-46.1)$ & EbpA (19), EbpC (27), EbpD (1), PigEBITS5 (2) \\
\hline Ruoqiang & $70 / 149$ & $47.0(38.9-55.1)$ & CS-1 (2), D (2), EbpA (5), EbpC (60), H (1) \\
\hline Total & $389 / 801$ & $48.6(45.1-52.0)$ & $\begin{array}{l}\text { CHC5 (2), CS-1 (5), CS-4 (20), CS-7 (3), CS-9 (1), D (17), EbpA (129), EbpC (168), } \\
\text { EbpD (5), H (2), PigEb4 (12), PigEBITS5 (19), WildBoar8 (3), XJP-II (2), XJP-III (1) }\end{array}$ \\
\hline
\end{tabular}

Genotypes detected in humans are in bold, and dominant genotypes are in italics.

TABLE 2 | Enterocytozoon bieneusi occurrence and genotype in pigs in China.

\begin{tabular}{|c|c|c|c|}
\hline Province & $\begin{array}{l}\text { No. of } \\
\text { positive/No. of } \\
\text { examined (\%) }\end{array}$ & Enterocytozoon bieneusi genotypes (n) & References \\
\hline Guangdong & $19 / 72(26.4 \%)$ & $E_{b p A^{a}}(1), \boldsymbol{E b p C}(17)$, GD1 (1) & Zou et al., 2018 \\
\hline Heilongjiang ${ }^{C}$ & $351 / 641(54.8 \%)$ & $\begin{array}{l}\text { CC-1 (2), CHN7/O (1), CS-1 (8), CS-1/EbpC (1), CS-2 (1), CS-3 (1), } \\
\text { CS-3/EbpA a (2), CS-4 (34), CS-5 (1), CS-6 (1), CS-7 (1), CS-8 (4), CS-10 (1), D } \\
\text { (20), EbpA a (37), EbpAa/EbpC (4), EbpAa/Henan-IV (1), EbpB (28), EbpB/EbpC } \\
\text { (1), EbpC (61), EbpC/Henan-IV (1), EbpC/O (30), EbpD (1), H (18), Henan-IV (6), } \\
\text { HLJ-I (2), HLJ-II (1), HLJ-III (1), HLJ-IV (1), LW1 (1), O (18), } \\
\text { PigEBITS5a/Henan-IV (1) }\end{array}$ & $\begin{array}{l}\text { Li et al., 2014a,c; Zhao et al., } 20 \\
\text { Wan et al., } 2016\end{array}$ \\
\hline Henan $^{\mathrm{b}}$ & $744 / 1372(54.2 \%)$ & $\begin{array}{l}\text { CHC5 (4), CM8 (11), EbpA a (154), EbpC (278), G (10), H (14), HN-1 (6), HN-2 } \\
\text { (2), HN-3 (1), HN-4 (1), Henan-III (1), LW1 (12), PigEBITS4a (24), PigEbITS5a } \\
\text { (17), XZP-II (1) }\end{array}$ & Wang et al., 2018a; Li W. et al., 2 \\
\hline Inner mongolia & $3 / 8(37.5 \%)$ & CHN7 (1), EbpC (1), O (1) & Li et al., 2014a,c \\
\hline Jilin $^{\mathrm{C}}$ & $145 / 330(43.9 \%)$ & 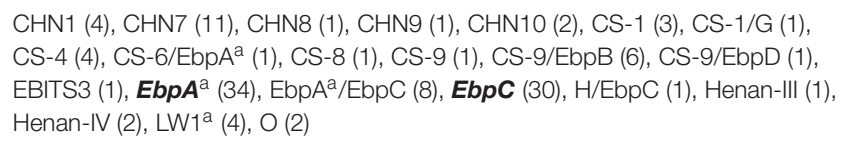 & $\begin{array}{l}\text { Li et al., 2014a,b,c; Wan et al., } 2( \\
\text { Zhang et al., } 2011\end{array}$ \\
\hline Liaoning $^{c}$ & $13 / 73(17.4 \%)$ & EbpB/EbpC (6) & Li et al., 2014c; Wan et al., 2016 \\
\hline Shaanxi & $442 / 560(78.9 \%)$ & 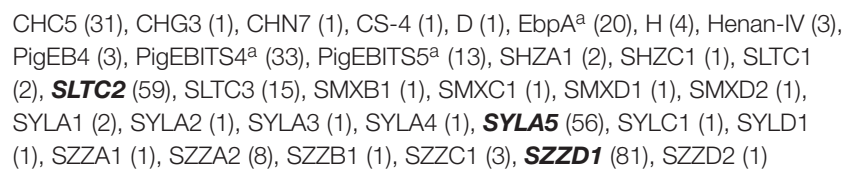 & Wang et al., 2018b \\
\hline Sichuan & $230 / 623(36.9 \%)$ & $\begin{array}{l}\text { CHC5 (10), D (1), EbpA }{ }^{a}(22), \text { EbpC (143), Henan-IV (24), PigEBITS4a (12), } \\
\text { PigEBITS5 (1), SH8a (6), WildBoar } 7 \text { (1), WildBoar 8 }{ }^{a} \text { (7), WildBoar } 11 \text { (1), SC02 } \\
\text { (1), SCT01 (1), SCTO2 (1) }\end{array}$ & Li et al., 2017; Luo et al., 2019 \\
\hline Tibet & $309 / 715(43.2 \%)$ & D (1), EbpC (302), I (2), XZP-I (1), XZP-\| (3) & Li W. et al., 2019 \\
\hline Yunnan & $59 / 200(29.5 \%)$ & $\begin{array}{l}\text { D (1), EbpA a (15), Ebpc (31), G (1), H (1), Henan-IV (6), PigEBITS5a (1), YN1 } \\
\text { (1), YN2 (2), YN3 (1) }\end{array}$ & Zou et al., 2018 \\
\hline Zhejiang & $47 / 124(37.9 \%)$ & CAF-1 (2), EbpA ${ }^{a}$ (2), EbpC (39), PigEBITS5a (2), ZJ1 (1), ZJ2 (1) & Zou et al., 2018 \\
\hline
\end{tabular}

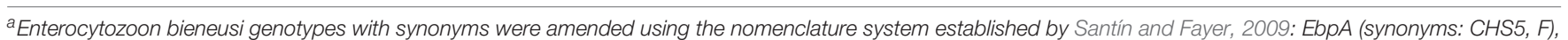

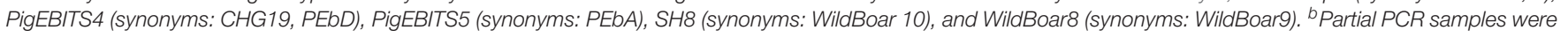
selected for sequencing. ' Partial PCR samples were not successfully sequenced. Dominant genotypes are in italics. 
Li W. et al., 2019; Zhao et al., 2014; Wan et al., 2016; Wang et al., 2018a; Zou et al., 2018; Luo et al., 2019). In China, Xinjiang Uygur Autonomous Region (hereafter referred to as Xinjiang) lies in inland Eurasia and has a typical half-arid/arid climate $\left(34^{\circ} 25^{\prime}-\right.$ $\left.48^{\circ} 10^{\prime} \mathrm{N}, 73^{\circ} 40^{\prime}-96^{\circ} 18^{\prime} \mathrm{E}\right)$. It is the largest provincial-level administrative region by land area and a historically important passage of the ancient silk road. Information on the occurrence of E. bieneusi in pigs in Xinjiang is scarce; therefore, this study was conducted to examine the occurrence of E. bieneusi in pigs in Xinjiang, China, and to assess the zoonotic transmission risk of this pathogen.

\section{MATERIALS AND METHODS}

\section{Ethics Statement}

Permission was obtained from animal owners or managers before collecting specimens, and no specific permits were required for the described field studies. All work involving animals was carried out in accordance with the Regulations for the Administration of Affairs Concerning Experimental Animals. The Research Ethics Committee of Henan Agricultural University reviewed and approved our study (approval no. LVRIAEC 2017-019).

\section{Fecal Specimen Collection, DNA Extraction, and Purification}

Eight hundred one fresh fecal specimens were collected from Duroc and Landrace pigs on seven large-scale intensive pig farms in Xinjiang between September 2017 and June 2018. Each sampled farm contained 10000-80000 pigs. All farms were visited on a single occasion. A veterinarian randomly collected the fecal specimens either from the rectum or from the internal portion of a stool sample on the ground avoid possible contamination from the specimen surface touched the ground. All specimens (approximately 5-30 g) were collected using sterile disposal latex gloves; marked with the date, age, and farm; stored in insulated boxes; and transferred to the laboratory. Collected specimens included 169 fecal specimens from pre-weaned suckling pigs ( $<20$ days old), 281 specimens from post-weaned suckling pigs (21-70 days old), 129 specimens from fattening pigs (71-180 days old), and 222 specimens from sows ( $>181$ days old).

Genomic DNA was directly extracted from the fecal specimens (approximately $200 \mathrm{mg}$ ) using the E.Z.N.A. ${ }^{\circledR}$ Stool DNA Kit (Omega Biotek Inc., Norcross, GA, United States) per the manufacturer's instructions with minor modifications. The extracted DNA was stored at $-20^{\circ} \mathrm{C}$ prior to polymerase chain reaction (PCR) analysis.

\section{PCR Amplification and Sequence Analysis}

Enterocytozoon bieneusi was identified via nested PCR amplification and sequencing of the ITS region of the rRNA gene. The primers and thermal cycle parameters used for the two PCR amplifications have been described previously (Buckholt et al., 2002). The outer primers were EBITS3 (5'-GGTCATAGGGATGAAGAG) and EBITS4
(5'-TTCGAGTTCTTTCGCGCTC), and the cycling parameters were 35 cycles of $94^{\circ} \mathrm{C}$ for $30 \mathrm{~s}, 57^{\circ} \mathrm{C}$ for $30 \mathrm{~s}$, and $72^{\circ} \mathrm{C}$ for $40 \mathrm{~s}$. The inner primers were EBITS 1 $\left(5^{\prime}\right.$-GCTCTGAATATCTATGGCT) and EBITS2.4 (5'ATCGCCGACGGATCCAAGTG), and the cycling parameters were 30 cycles of $94^{\circ} \mathrm{C}$ for $30 \mathrm{~s}, 55^{\circ} \mathrm{C}$ for $30 \mathrm{~s}$, and $72^{\circ} \mathrm{C}$ for $40 \mathrm{~s}$. The $2 \times$ EasyTaq PCR SuperMix (TransGene Biotech Co., Beijing, China) was used for PCR amplification. All PCR assays included both a positive control (DNA from dairy cattle-derived genotype I) and a negative control (distilled water). PCR amplification was repeated twice for each specimen.

Positive secondary PCR products ( $\sim 390 \mathrm{bp})$ were sequenced by GENEWIZ (Suzhou, China), and all products were sequenced in both directions to ensure accurate sequencing results. ClustalX $2.1^{1}$ was used to align the resulting DNA sequences. Sequences obtained were aligned with reference sequences downloaded from the National Center for Biotechnology Information ${ }^{2}$ to determine genotypes. The nucleotide sequences obtained in the present study were submitted to GenBank ${ }^{3}$ under accession numbers MK778892-K778899 and MK778901-MK778907.

\section{Phylogenetic and Statistical Analysis}

Bayesian inference (BI) and Monte Carlo Markov chain methods were used to construct phylogenetic trees in MrBayes,

\footnotetext{
${ }^{1}$ http://www.clustal.org/

${ }^{2}$ https://www.ncbi.nlm.nih.gov/

${ }^{3} \mathrm{https} / / /$ www.ncbi.nlm.nih.gov/genbank/
}

TABLE 3 | Enterocytozoon bieneusi occurrence and genotypes in pigs of different ages.

\begin{tabular}{|c|c|c|c|}
\hline Age (days) & $\begin{array}{l}\text { No. positive/No. } \\
\text { specimens }\end{array}$ & $\%(95 \mathrm{Cl})$ & $\begin{array}{l}\text { Enterocytozoon } \\
\text { bieneusi } \\
\text { genotypes (n) }\end{array}$ \\
\hline Pre-weaned & $60 / 169$ & $35.5(28.2-42.8)$ & $\begin{array}{l}\text { EbpC (24), EbpA } \\
\text { (12), D (11), PigEb4 } \\
\text { (5), PigEBITS5 (4), } \\
\text { CS-4 (3), } \\
\text { WildBoar8 (1) }\end{array}$ \\
\hline Post-weaned & $217 / 281$ & $77.2(72.3-82.2)$ & $\begin{array}{l}\text { EbpC (105), EbpA } \\
\text { (64), PigEBITS5 } \\
\text { (11), CS-4 (9), D (6), } \\
\text { PigEb4 (5), EbpD } \\
\text { (4), CS-1 (4), CS-7 } \\
\text { (3), WildBoar8 (2), } \\
\text { CHC5 (1), H (1), } \\
\text { XJP-1 (2) }\end{array}$ \\
\hline Fattening pigs & $87 / 129$ & $67.4(59.2-75.6)$ & $\begin{array}{l}\text { EbpA (46), Ebpc } \\
\text { (26), CS-4 (7), } \\
\text { PigEBITS5 (3), } \\
\text { CHC5 (1), CS-1 (1), } \\
\text { CS-9 (1), H (1), } \\
\text { XJP-2 (1) }\end{array}$ \\
\hline Sow & $25 / 222$ & $11.3(7.1-15.5)$ & $\begin{array}{l}\text { EbpC (13), EbpA } \\
\text { (7), PigEb4 (2), } \\
\text { CS-4 (1), EbpD (1), } \\
\text { PigEBITS5 (1) }\end{array}$ \\
\hline
\end{tabular}

Genotypes detected in humans are in bold and dominant genotypes were in italics. 


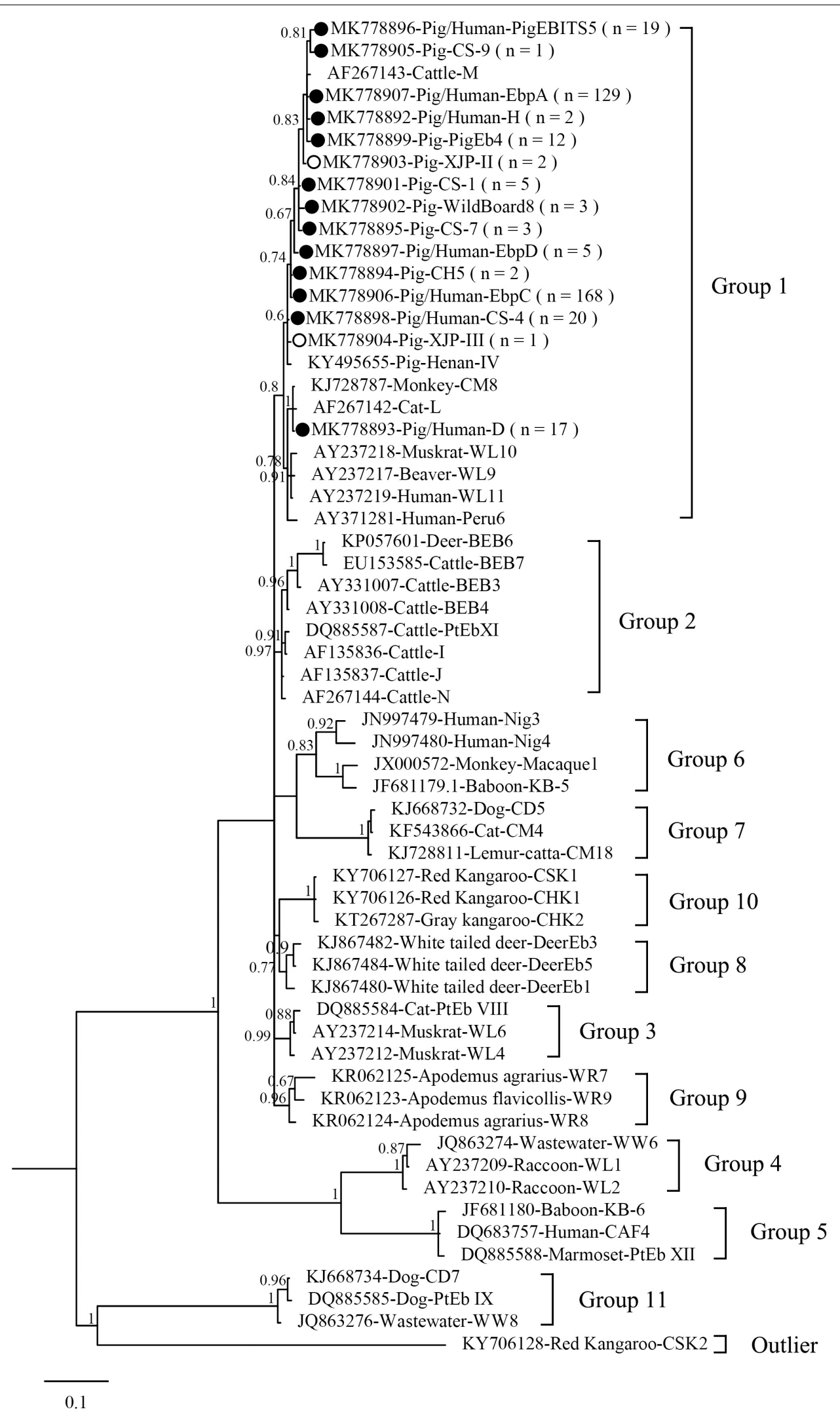

FIGURE 1 | Phylogenetic tree based on Bayesian analysis of the ITS sequences. Statistically significant posterior probabilities are indicated on the branches. Known and novel Enterocytozoon bieneusi genotypes identified in the present study are indicated by filled and hollow circles, respectively. 
version 3.2.6 ${ }^{4}$. The posterior probability values were calculated by running 1,000,000 generations. A 50\% majority-rule consensus tree was constructed from the final $75 \%$ of the trees generated via BI. Analyses were run three times to ensure convergence and insensitivity to priors.

The Statistical Package for the Social Sciences (SPSS, version 22.0, available at https://www.ibm.com) was used for the statistical analyses, including Fisher's exact test and 95\% confidence intervals. Differences with $p<0.05$ were considered significant.

\section{RESULTS AND DISCUSSION}

In the present study, the overall $E$. bieneusi infection rate in pigs was $48.6 \%(389 / 801)$ (Table 1), which was higher than most previously reported rates from Chinese provinces, including Guangdong (26.4\%, 19/72) (Zou et al., 2018), Inner Mongolia (37.5\%, 3/8) (Li et al., 2014a,c), Jilin (43.9\%, 145/330) (Zhang et al., 2011; Li et al., 2014a,b,c; Wan et al., 2016), Liaoning $(17.4 \%, 13 / 73)$ (Li et al., 2014c; Wan et al., 2016), Sichuan (36.9\%, 230/623) (Li et al., 2017; Luo et al., 2019), Tibet (43.2\%, 309/715) (Li W. et al., 2019), Yunnan (29.5\%, 59/200) and Zhejiang $(37.9 \%, 47 / 124)$ (Zou et al., 2018), but lower than that from Shaanxi (78.9\%, 442/560) (Wang et al., 2018b; Table 2). The differences in E. bieneusi infection rates may be partially attributed to differences in feeding densities, comparisons with cages that had lower pig densities, and greater opportunities for E. bieneusi transmission among animals in high densely packed cages (Wang et al., 2018b).

The E. bieneusi infection rates significantly differed among the collection sites $\left(\chi^{2}=75.720, d f=6, p<0.01\right)$. The highest infection rate was found on a farm from Shaya $(73.0 \%, 73 / 100)$ (Table 1). The E. bieneusi infection rate also differed significantly among age groups $\left(\chi^{2}=246.015, d f=3, p<0.01\right)$. Postweaned pigs had the highest infection rate $(77.2 \%, 217 / 281)$, while adult pigs (sows) had the lowest infection rate $(11.3 \%$, 25/222) (Table 3). Most previous studies included information on E. bieneusi for different age groups of pigs in China. High E. bieneusi infection rates were found in pre-weaned and postweaned pigs from Heilongjiang (78.4\%, 87/111; 70.4\%, 50/71), Jilin (68.8\%, 22/32; 74.6\%, 53/71), Zhejiang (54.7\%, 76/139), and Henan Provinces (54.2\%, 137/253) (Li et al., 2014b, Li W. et al., 2019; Wan et al., 2016; Zou et al., 2018). The higher prevalence among post-weaned pigs may have been due to lower immunity and stress from early wean (Wang et al., 2018b). However, high E. bieneusi infection rates were found in fattening pigs from Liaoning (100\%, 3/3), Jilin (35.7\%, 20/56), Tibet (75.5\%, 173/229) and Yunnan Provinces $(21 \%, 42 / 200$ ) (Wan et al., 2016; Zou et al., 2018; Li W. et al., 2019). The differences in E. bieneusi infection rates in these age groups may be partially attributed to differences in geoecology, rearing conditions, animal husbandry, and feeding densities.

From 389 positive specimens, 15 E. bieneusi genotypes were identified from nucleotide sequences via ITS-PCR. These

${ }^{4} \mathrm{http}: / /$ mrbayes.sourceforge.net/ included 13 known genotypes (CHC5, CS-1, CS-4, CS-9, CS-7, D, EbpA, EbpC, EbpD, H, PigEb4, PigEBITS5, and WildBoard8) and 2 novel genotypes (named XJP-II and XJP-III; Table 1). $\operatorname{EbpC}(43.2 \%, 168 / 389)$ and $\operatorname{EbpA}(33.2 \%, 129 / 389)$ were the predominant genotypes in pigs in Xinjiang (Table 1). The dominant genotypes also varied across sample regions. Genotype EbpC was dominant in Marabishi, Alaer, Changji, and Ruoqiang; genotype EbpA was dominant in Yarkant and Baicheng; and genotype CS-4 was dominant and identified only in Shaya (Table 1). In addition, the E. bieneusi genotype distribution differed among ages. Genotype EbpC was predominant in preweaned, post-weaned, and adult pigs, while EbpA predominated in fattening pigs. These results were similar to those reported for Guangdong, Henan, Jilin, Sichuan, Tibet, Yunnan and Zhejiang Provinces in China, where reported genotypes EbpC and EbpA were predominant in pigs (Li et al., 2014a,b, 2017, Li W. et al., 2019; Zhao et al., 2014; Wan et al., 2016; Wang et al., 2018a; Zou et al., 2018; Luo et al., 2019; Table 2).

Of the 120 E. bieneusi genotypes reported in pigs worldwide (Sak et al., 2008; Němejc et al., 2014; Fiuza et al., 2015; Prasertbun et al., 2017; Wang et al., 2018a,b), over 80 have been reported in pigs in China (Wan et al., 2016; Wang et al., 2018b,c; Li W. et al., 2019). Fifteen genotypes were identified in the present study, of which, six (CS-4, D, EbpA, EbpC, H and PigEBITS5) have been identified in humans. Genotypes CS-4, D, EbpA, EbpC, and H were identified in children and HIV/AIDS patients from China (Wang et al., 2013; Yang et al., 2014; Liu et al., 2017). These results suggest that pigs play an important role in transmitting E. bieneusi to humans and other animals.

Figure 1 shows the phylogeny of the ITS sequences from the 15 genotypes identified in the present study, and all genotypes identified were classified in Group 1. Accumulating evidence suggests that genotypes in Group 1 have significant zoonotic importance but no strong host specificity (Wan et al., 2016; Li and Xiao, 2019; Li W. et al., 2019). Although direct evidence linking human infections to E. bieneusi of animal origin is lacking, direct contact with pigs or with a water supply contaminated by pig waste are considered significant risk factors for zoonotic transmission (Cama et al., 2007).

\section{CONCLUSION}

This study revealed that E. bieneusi is common in pigs in Xinjiang, China. Thirteen known genotypes and two novel genotypes (XIP-II and XIP-III) were classified in Group 1, and showed six of the 15 identified genotypes have been found in humans, indicating that pigs may be reservoirs for zoonotic transmission of human microsporidiosis. These findings extend the knowledge of the E. bieneusi distribution among pigs in China.

\section{DATA AVAILABILITY STATEMENT}

The datasets generated for this study can be found in GenBank under the accession numbers MK778892-K778899 and MK778901-MK778907. 


\section{ETHICS STATEMENT}

Permission was obtained from animal owners or managers before collecting specimens, and no specific permits were required for the described field studies. All work involving animals was carried out in accordance with the Regulations for the Administration of Affairs Concerning Experimental Animals. The Research Ethics Committee of Henan Agricultural University reviewed and approved our study (approval no. LVRIAEC 2017-019).

\section{AUTHOR CONTRIBUTIONS}

MQ and L-XZ designed the study. YZ, Y-XJ, J-MX, D-YT, A-YZ, and $\mathrm{BJ}$ collected and analyzed the specimens. D-FL and Z-HC analyzed the data. D-FL, MQ, and L-XZ wrote the manuscript. All authors read and approved the final manuscript.

\section{REFERENCES}

Buckholt, M. A., Lee, J. H., and Tzipori, S. (2002). Prevalence of Enterocytozoon bieneusi in swine: an 18-month survey at a slaughterhouse in Massachusetts. Appl. Environ. Microbiol. 68, 2595-2599. doi: 10.1128/AEM.68.5.2595-2599. 2002

Cama, V. A., Pearson, J., Cabrera, L., Pacheco, L., Gilman, R., Meyer, S., et al. (2007). Transmission of Enterocytozoon bieneusi between a child and guinea pigs. J. Clin. Microbiol. 45, 2708-2710. doi: 10.1128/JCM.00725-07

del Aguila, C., Lopez-Velez, R., Fenoy, S., Turrientes, C., Cobo, J., Navajas, R., et al. (1997). Identification of Enterocytozoon bieneusi spores in respiratory samples from an AIDS patient with a 2-year history of intestinal microsporidiosis. J. Clin. Microbiol. 35, 1862-1866.

Deng, L., Li, W., Zhong, Z., Chai, Y., Yang, L., Zheng, H., et al. (2018). Molecular characterization and new genotypes of Enterocytozoon bieneusi in pet chipmunks (Eutamias asiaticus) in Sichuan province, China. BMC Microbiol. 18:37. doi: 10.1186/s12866-018-1175-y

Fiuza, V. R. S., Oliveira, F. C. R., Fayer, R., and Santín, M. (2015). First report of Enterocytozoon bieneusi in pigs in Brazil. Parasitol. Int. 64, 18-23. doi: 10.1016/ j.parint.2015.01.002

Galván-Díaz, A. L., Magnet, A., Fenoy, S., Henriques-Gil, N., Haro, M., Gordo, F. P., et al. (2014). Microsporidia detection and genotyping study of human pathogenic E. bieneusi in animals from Spain. PLoS One 9:e92289. doi: 10.1371/ journal.pone.0092289

Henriques-Gil, N., Haro, M., Izquierdo, F., Fenoy, S., and del Águila, C. (2010). Phylogenetic approach to the variability of the microsporidian Enterocytozoon bieneusi and its implications for inter- and intrahost transmission. Appl. Environ. Microbiol. 76, 3333-3342. doi: 10.1128/AEM.03026-09

Karim, M. R., Wang, R., Dong, H., Zhang, L., Li, J., Zhang, S., et al. (2014). Genetic polymorphism and zoonotic potential of Enterocytozoon bieneusi from nonhuman primates in China. Appl. Environ. Microbiol. 80, 1893-1898. doi: 10.1128/AEM.03845-13

Li, W., Deng, L., Wu, K., Huang, X., Song, Y., and Su, H. (2017). Presence of zoonotic Cryptosporidium scrofarum, Giardia duodenalis assemblage A and Enterocytozoon bieneusi genotypes in captive Eurasian wild boars (Sus scrofa) in China: potential for zoonotic transmission. Parasit Vect. 10:10. doi: 10.1186/ s13071-016-1942-2

Li, W., Diao, R., Yang, J., Xiao, L., Lu, Y., Li, Y., et al. (2014a). High diversity of human-pathogenic Enterocytozoon bieneusi genotypes in swine in northeast China. Parasitol. Res. 113, 1147-1153. doi: 10.1007/s00436-0143752-9

Li, W., Li, Y., Li, W., Yang, J., Song, M., Diao, R., et al. (2014b). Genotypes of Enterocytozoon bieneusi in livestock in China: high prevalence and zoonotic potential. PLoS One 9:e97623. doi: 10.1371/journal.pone.0097623

Li, W., Tao, W., Jiang, Y., Diao, R., Yang, J., and Xiao, L. (2014c). Genotypic distribution and phylogenetic characterization of Enterocytozoon bieneusi in

\section{FUNDING}

This work was supported in part by the National Natural Science Foundation of China (31860699, 31702227, and 31660712) and the Program for Young and Middle-aged Leading Science, Technology, and Innovation of Xinjiang Production \& Construction Group (2018СB034). The sponsors played no roles in the study design or in the collection, analysis, or interpretation of the data, in writing the report, or in the decision to submit the article for publication.

\section{ACKNOWLEDGMENTS}

We thank Traci Raley, MS, ELS, from Liwen Bianji, Edanz Editing China (www.liwenbianji.cn/ac) for editing a draft of this manuscript.

diarrheic chickens and pigs in multiple cities, China: potential zoonotic transmission. PLoS One 9:e108279. doi: 10.1371/journal.pone.0108279

Li, W., Feng, Y., and Santin, M. (2019). Host specificity of Enterocytozoon bieneusi and public health implications. Trends Parasitol. 35, 436-451. doi: 10.1016/j.pt. 2019.04.004

Li, D., Zheng, S., Zhou, C., Karim, M. R., Wang, L., Wang, H., et al. (2019). Multilocus typing of Enterocytozoon bieneusi in pig reveals the high prevalence, zoonotic potential, host adaptation and geographical segregation in China. J. Eukaryot. Microbiol. 66, 707-718. doi: 10.1111/jeu.12715

Li, W., and Xiao, L. (2019). Multilocus sequence typing and population genetic analysis of Enterocytozoon bieneusi: host specificity and its impacts on public health. Front. Genet. 10:307. doi: 10.3389/fgene.2019.00307

Liu, H., Jiang, Z., Yuan, Z., Yin, J., Wang, Z., Yu, B., et al. (2017). Infection by and genotype characteristics of Enterocytozoon bieneusi in HIV/AIDS patients from Guangxi Zhuang autonomous region, China. BMC Infect. Dis. 17:684. doi: 10.1186/s12879-017-2787-9

Luo, R., Xiang, L., Liu, H., Zhong, Z., Liu, L., Deng, L., et al. (2019). First report and multilocus genotyping of Enterocytozoon bieneusi from Tibetan pigs in southwestern China. Parasite 26:24. doi: 10.1051/parasite/2019021

Matos, O., Lobo, M. L., and Xiao, L. (2012). Epidemiology of Enterocytozoon bieneusi infection in humans. J. Parasitol. Res. 2012:981424. doi: 10.1155/2012/ 981424

Němejc, K., Sak, B., Květoňová, D., Hanzal, V., Janiszewski, P., Forejtek, P., et al. (2014). Prevalence and diversity of Encephalitozoon spp. and Enterocytozoon bieneusi in wild boars (Sus scrofa) in Central Europe. Parasitol. Res. 113, 761-767. doi: 10.1007/s00436-013-3707-6

Prasertbun, R., Mori, H., Pintong, A. R., Sanyanusin, S., Popruk, S., Komalamisra, C., et al. (2017). Zoonotic potential of Enterocytozoon genotypes in humans and pigs in Thailand. Vet. Parasitol. 233, 73-79. doi: 10.1016/j.vetpar.2016.12.002

Sak, B., Kvác, M., Hanzlíková, D., and Cama, V. (2008). First report of Enterocytozoon bieneusi infection on a pig farm in the Czech Republic. Vet. Parasitol. 153, 220-224. doi: 10.1016/j.vetpar.2008.01.043

Sak, B., Kváč, M., Kučerová, Z., Květoňová, D., and Saková, K. (2011). Latent microsporidial infection in immunocompetent individuals-a longitudinal study. PLoS Negl. Trop. Dis. 5:e1162. doi: 10.1371/journal.pntd.0001162

Santín, M. and Fayer, R. (2009). Enterocytozoon bieneusi genotype nomenclature based on the internal transcribed spacer sequence: a consensus. J. Eukaryot. Microbiol. 56, 34-38. doi: 10.1111/j.1550-7408.2008.00380.x

Santín, M., and Fayer, R. (2011). Microsporidiosis: Enterocytozoon bieneusi in domesticated and wild animals. Res. Vet. Sci. 90, 363-371. doi: 10.1016/j.rvsc. 2010.07.014

Stentiford, G. D., Becner, J. J., Weiss, L. M., Keeling, P. J., Didier, E. S., Williams, B. A. P., et al. (2016). Microsporidia-emergent pathogens in the global food chain. Trends Parasitol. 32:657. doi: 10.1016/j.pt.2016.06.002

Wan, Q., Lin, Y., Mao, Y., Yang, Y., Li, Q., Zhang, S., et al. (2016). High prevalence and widespread distribution of zoonotic Enterocytozoon bieneusi genotypes in 
swine in Northeast China: implications for public health. J. Eukaryot. Microbiol. 63, 162-170. doi: 10.1111/jeu.12264

Wang, H., Zhang, Y., Wu, Y., Li, J., Qi, M., Li, T., et al. (2018a). Occurrence, molecular characterization, and assessment of zoonotic risk of Cryptosporidium spp., Giardia duodenalis, and Enterocytozoon bieneusi in Pigs in Henan, Central China. J. Eukaryot. Microbiol. 65, 893-901. doi: 10.1111/jeu.12634

Wang, S. S., Li, J. Q., Li, Y. H., Wang, X. W., Fan, X. C., Liu, X., et al. (2018b). Novel genotypes and multilocus genotypes of Enterocytozoon bieneusi in pigs in northwestern China: a public health concern. Infect. Genet. Evol. 63, 89-94. doi: 10.1016/j.meegid.2018.05.015

Wang, S. S., Wang, R. J., Fan, X. C., Liu, T. L., Zhang, L. X., and Zhao, G. H. (2018c). Prevalence and genotypes of Enterocytozoon bieneusi in China. Acta Trop. 183, 142-152. doi: 10.1016/j.actatropica.2018.04.017

Wang, H. Y., Qi, M., Sun, M. F., Li, D. F., Wang, R. J., Zhang, S. M., et al. (2019). Prevalence and population genetics analysis of Enterocytozoon bieneusi in dairy cattle in China. Front. Microbiol. 10:1399. doi: 10.3389/fmicb.2019. 01399

Wang, L., Xiao, L., Duan, L., Ye, J., Guo, Y., Guo, M., et al. (2013). Concurrent infections of Giardia duodenalis, Enterocytozoon bieneusi, and Clostridium difficile in children during a cryptosporidiosis outbreak in a pediatric hospital in China. PLoS Negl. Trop. Dis. 7:e2437. doi: 10.1371/journal.pntd.000 2437

Yang, J., Song, M., Wan, Q., Li, Y., Lu, Y., Jiang, Y., et al. (2014). Enterocytozoon bieneusi genotypes in children in Northeast China and assessment of risk of zoonotic transmission. J. Clin. Microbiol. 52, 4363-4367. doi: 10.1128/JCM. 02295- 14

Zhang, X., Wang, Z., Su, Y., Liang, X., Sun, X., Peng, S., et al. (2011). Identification and genotyping of Enterocytozoon bieneusi in China. J. Clin. Microbiol. 49, 2006-2008. doi: 10.1128/JCM.00372-11

Zhao, W., Zhang, W., Yang, F., Cao, J., Liu, H., Yang, D., et al. (2014). High prevalence of Enterocytozoon bieneusi in asymptomatic pigs and assessment of zoonotic risk at the genotype level. Appl. Environ. Microbiol. 80, 3699-3707. doi: 10.1128/aem.00807-14

Zou, Y., Hou, J. L., Li, F. C., Zou, F. C., Lin, R. Q., Ma, J. G., et al. (2018). Prevalence and genotypes of Enterocytozoon bieneusi in pigs in southern China. Infect. Genet. Evol. 66, 52-56. doi: 10.1016/j.meegid.2018.09.006

Conflict of Interest: The authors declare that the research was conducted in the absence of any commercial or financial relationships that could be construed as a potential conflict of interest.

Copyright (c) 2019 Li, Zhang, Jiang, Xing, Tao, Zhao, Cui, Jing, Qi and Zhang. This is an open-access article distributed under the terms of the Creative Commons Attribution License (CC BY). The use, distribution or reproduction in other forums is permitted, provided the original author(s) and the copyright owner(s) are credited and that the original publication in this journal is cited, in accordance with accepted academic practice. No use, distribution or reproduction is permitted which does not comply with these terms. 